\title{
Efecto del sistema binominal en el número de candidatos y de partidos en elecciones legislativas en Chile, 1989-2001
}

José Miguel CABEZAS y Patricio NAVIA

\section{Introducción}

$\mathrm{L}$ os sistemas electorales a menudo son diseñados para intentar influir en la forma en que evoluciona el sistema de partidos políticos. Así, cuando el sistema de partidos es considerado demasiado inestable, se pueden adoptar reformas al sistema electoral que tiendan a consolidar partidos políticos más estables y duraderos. De la misma forma, cuando los partidos políticos que existen en el país no representan adecuadamente los diferentes grupos y corrientes políticas que prevalecen en la sociedad, los sistemas electorales pueden ser modificados para facilitar el ingreso de nuevos partidos que contribuyan a alterar la composición del sistema de partidos. En el caso de Chile, como discutimos en este artículo, al fin de la dictadura se adoptó un nuevo sistema electoral. Aunque fue adoptado con la intención de reducir el número de partidos políticos que existían antes del quiebre de la democracia en 1973, el sistema binominal no ha contribuido a ese objetivo desde que fue utilizado por primera vez en 1989.

En este artículo demostramos que el número de partidos y de candidatos se mantuvo estable entre 1989 y el 2001. Argumentamos también que la tasa considerablemente alta de diputados que buscan la reelección ayu-

1. Agradecemos los comentarios de Cristóbal Aninat, Juan Esteban Montes, José Miguel Sandoval. Una versión anterior de este trabajo fue publicada como Documento de Trabajo No 3, año 1 (2005) del ICSO de la Universidad Diego Portales. 
da a mantener relativamente bajo el número efectivo de partidos y candidatos en los 60 distritos de la Cámara de Diputados en el país. Por el contrario, debido a que no había titulares que buscaban la reelección, el número de partidos y candidatos en las parlamentarias de 1989 fue superior al observado en elecciones posteriores. Este artículo está dividido en tres secciones. Primero, discutimos el origen del sistema binominal, los argumentos utilizados para justificar su adopción en 1989 y sus principales características. En segundo término, explicamos la metodología utilizada en este estudio, enfatizando nuestro interés en medir tanto el número efectivo de partidos políticos como el número efectivo de candidatos en las 4 elecciones legislativas celebradas entre 1989 y el 2001. En tercer lugar, discutimos los resultados, destacando la evidencia que nos permite concluir que el número efectivo de partidos y candidatos por distrito no ha disminuido significativamente a través de los años, manteniéndose en una cantidad superior a lo esperado por los diseñadores electorales. Argumentamos, no obstante, que la presencia de candidatos que buscan la reelección a la Cámara de Diputados tiene un efecto importante en contribuir a la disminución del número efectivo de candidatos y partidos en las elecciones chilenas celebradas con posterioridad a 1989. Finalmente, sugerimos que la presencia de más candidatas mujeres ha contribuido a disminuir el número de partidos y candidatos en las contiendas parlamentarias distritales desde 1989 hasta el 2001.

\section{Origen y características del sistema binominal}

Los críticos del sistema binominal a menudo han resaltado el hecho de que tanto la magnitud de distrito (2 escaños por distrito) como el mapa electoral fueron diseñados para favorecer a los sectores más propensos a apoyar a los partidos políticos que se identificaban con la saliente dictadura militar (Sigmund, 1993: 185; Garretón, 1991; Nohlen, 1994: 238-242; Collier y Sater, 1996: 381; Siavelis, 1997a: 34; 1997b; 2000: 34-35; y Portales, 2000: 39). Los encargados de diseñar el sistema electoral en el régimen de Pinochet estaban interesados en idear mecanismos que perpetuaran, o mejoraran, las posibilidades de que sus adherentes pudieran tener una buena representación en la Cámara de Diputados a escogerse en diciembre de 1989. Ya que posee una magnitud de distrito menor al de antes de 1973, algunos lo han descrito como 'semi-mayoritario' (Fernández 1998; Nohlen 1994: 238). Por otro lado, debido a la reducida magnitud de distrito, el sistema a menudo es asociado con la reducción en el número de partidos e incluso con la consolidación de un sistema bipartidista (Libertad y Desarrollo, 2001).

La discusión sobre el verdadero origen del sistema binominal no ha estado exenta de polémica. Aunque la Constitución establecía que cada región escogería dos senadores en forma simultánea, no estipuló explícitamente cómo se escogerían ambos escaños. El artículo 45 de la Constitución señalaba, en su versión original, que "a cada región corres- 
ponderá elegir dos senadores, en la forma que determine la ley orgánica constitucional respectiva". Si bien es cierto, el referido artículo no señala que cada región debería constituir un distrito electoral único o que se debía utilizar un sistema de representación proporcional para asignar ambos escaños (como bien subraya Pastor, 2004), el constituyente no tenía en mente un sistema mayoritario de lista cerrada (los dos escaños para el partido ganador) ni pensaba subdividir las regiones en más de un distrito (particularmente tomando en cuenta que existen regiones escasamente pobladas donde la comuna capital regional agrupa a la mayoría absoluta del electorado). Más bien, al promulgar la Constitución de 1980, el gobierno militar ya parecía tener preferencias claras por el sistema binominal. De hecho, el gobierno militar alegó que el sistema binominal "fue prácticamente consagrado por la propia Constitución para la elección de Senadores" (Historia de la Ley 18.799, 268).

Pero, formalmente, el sistema binominal fue recién adoptado para el Senado en la Ley Orgánica Constitucional sobre Votaciones Populares y Escrutinios (LOC 18.700) publicada en el Diario Oficial el 6 de mayo de $1988^{2}$. Más tarde, la Ley 18.799 (que modificó la LOC 18.700), promulgada el 26 de mayo de 1989, lo consagró como el sistema electoral a ser utilizado para las elecciones de la Cámara de Diputados. Una revisión cuidadosa de la Historia de la Ley 18.799 deja ver la preocupación tanto del Ejecutivo, que envió el proyecto inicial, como de la Junta de Gobierno (que ejercía de Poder Legislativo a través de sus comisiones legislativas) con el tamaño de distrito -conocido técnicamente como magnitud de distrito- que debía adoptarse para las elecciones de la Cámara de Diputados. De hecho, tan importante era esta consideración que el tamaño de los distritos fue conscientemente omitido de la LOC 18.700. Aunque la ley fue aprobada en abril de 1988, los mecanismos que rigen la forma en que se escogen los miembros de la Cámara de Diputados fueron promulgados después que se verificaron los resultados del plebiscito de 1988. No obstante, cuando el presidente Pinochet envió su mensaje a la Comisión Legislativa que debía abocarse a discutir la fórmula electoral para la composición de la Cámara de Diputados en agosto de 1988 (Historia de la Ley 18.799), dos meses antes del plebiscito, la intención del Ejecutivo era clara a favor de un sistema de dos escaños por distrito. El mensaje del Ejecutivo así lo hace evidente:

La iniciativa tiene por finalidad establecer un sistema electoral mayoritario, que dé expresión, fundamentalmente, a las grandes corrientes de opinión, que tenga un cierto efecto reductivo en el número de partidos, que no reitere la nefasta experiencia electoral y partidista del pasado, que ofrezca claridad al elector sobre el significado y consecuencias de su voto y que introduzca el pragmatismo en las decisiones en bien del país, favoreciendo la moderación de todos los actores políticos. Por lo anterior, el proyecto establece un sistema electoral uniforme que se aplique simultáneamente -en unas mismas elecciones, ya que así se desprende del texto

2. Para revisar una copia de la ley electoral ir a: http://www.servel.cl/servel/ index.aspx?channel $=125$ 
de la Constitución Política- y que permita elegir pocos diputados por distritos, concretamente dos en cada uno de ellos (Historia de la Ley 18.799, 39, el destacado es nuestro).

En el informe técnico que se entregó a la comisión de la Junta, junto al mensaje del Ejecutivo se repetía la explicación para justificar la elección de un sistema de dos escaños por distrito. Las posturas de las diferentes instancias legislativas del gobierno militar parecían coincidir en su preferencia por un sistema electoral que se alejara de la representatividad proporcional del sistema utilizado hasta antes de 1973. La preferencia por un sistema que favoreciera la moderación de los partidos convertía a los sistemas de representación proporcional en una opción poco deseada para la nueva institucionalidad democrática que habría de tener el país. Pero a la vez, el gobierno militar rechazaba la adopción de un sistema electoral mayoritario que indujera a los diferentes partidos a alinearse en dos grandes bloques. El mismo documento argumentaba en contra de los sistemas proporcionales al reflexionar de la siguiente forma:

¿Es indiferente para el país tropezar nuevamente con las situaciones electorales y partidistas de la década anterior a 1973, caracterizada por una competencia absurda por ser y parecer cada vez más extremista? ¿No es necesario acaso superar los excesos de ideologismos y abrir camino a un mayor pragmatismo y a una mejor coexistencia entre diferentes perspectivas y puntos de vista? En fin, ¿no es indispensable que el sistema, si no cree, por lo menos favorezca la moderación en los planteamientos políticos y dé lugar, de una vez por todas, a que el protagonismo de la política chilena lo tengan las posiciones más moderadas? (Historia de la Ley 18.799, 48)

Así, el gobierno militar rechazaba la adopción de un sistema de representación proporcional basándose en la experiencia de la democracia chilena del periodo pre-1973. La preocupación por diseñar un sistema que favoreciera la moderación parecía ser el componente central en la lógica que guió la adopción del sistema binominal. El gobierno militar se oponía tajantemente a la adopción de un sistema electoral que facilitara la existencia de múltiples partidos políticos. La reducción en el número de partidos y la preferencia por privilegiar la representación de los partidos políticos que tuvieran posturas más moderadas pareció ser la preocupación central al momento de escoger el nuevo sistema electoral que tendría el país. Al argumentar contra la representación proporcional que existía en Chile hasta 1973, el Informe Técnico señalaba que los sistemas proporcionales:

Cualquiera que sea la modalidad que adopten y por otros mecanismos con que se complementen, conducen fatalmente al multipartidismo excesivo y al fomento de ideologismos, que detienen el progreso nacional, desvían la atención de los reales problemas, confunden a la opinión pública con seudo soluciones, meramente intelectuales, sin arraigo serio en la realidad de la naturaleza del hombre y de la sociedad, ni en sus imperiosas necesidades, favoreciendo la demagogia al impedir al pueblo discernir sobre las cuestiones fundamentales (Historia de la Ley $18.799,49)$. 
El profundo rechazo a los sistemas de representación proporcional queda claramente establecido en diferentes y repetidos párrafos en el documento. Evidentemente, el gobierno militar rechazaba la posibilidad de adoptar un sistema electoral conducente a reproducir el sistema político pluripartidista que existía en el país hasta antes de 1973. La dictadura quería un sistema que fomentara la formación de dos grandes partidos o bloques políticos. Pero aunque lo natural hubiera sido la adopción de un sistema mayoritario, el gobierno militar parecía igualmente decidido a rechazar esa posibilidad. De hecho, el Informe Técnico rechazó la adopción de un sistema uninominal, de un diputado por distrito, y de un sistema proporcional de tres escaños por distrito:

Con atención a que ella daría paso a la posesión de la Cámara por una mayoría incontrarrestable, a la cual no podría ponerse freno con ningún quórum constitucional. Asimismo, se descarta la elección de tres diputados por distrito, toda vez que un sistema tal permite ratificar la relación electoral que ya experimentamos, en que más de los dos tercios se alejan del centro... (Historia de la Ley 18.799, 51).

La determinación en contra de los sistemas de representación proporcional y la oposición a un sistema verdaderamente mayoritario se mantuvieron durante todo el proceso legislativo en el año 1988 y 1989. Si bien la dictadura se oponía a los sistemas de representación proporcional, tampoco favorecía la adopción de un sistema mayoritario que fuera conducente a la formación y consolidación de dos grandes bloques políticos. Al presentar las modificaciones a la LOC para ser aprobadas, meses después del plebiscito de 1988, la Cuarta Comisión Legislativa de la Junta entregó un documento explicando sus motivos para adoptar pequeños cambios a la legislación inicialmente enviada por el Ejecutivo, y justificando la fórmula de dos escaños por distrito de la siguiente forma:

Considerando la necesidad que tiene el país de salir, en definitiva, del subdesarrollo político, económico y social, se estima del todo conveniente configurar un sistema que asegure, por una parte, la gobernabilidad del país y la eficiencia del Poder Legislativo y, por la otra, la representación de las minorías sin el peligro del bipartidismo (Historia de la Ley 18.799, 267).

Y con esa lógica se confirmó la adopción del sistema binominal. Aduciendo incluso a razones de coherencia constitucional, el texto de la Cuarta Comisión Legislativa justificó la adopción del sistema binominal de la siguiente forma:

Es necesario tener presente que el sistema mayoritario binominal fue prácticamente consagrado por la propia Constitución para la elección de Senadores, y que el mismo es más afín con el sistema presidencial previsto en la Carta Fundamental, al contrario de lo que ocurre con los sistemas proporcionales, que resultan más apropiados para los sistemas políticos de carácter parlamentario (Historia de la Ley 18.799, 268). 
Así, el diseñador electoral optó por un sistema que facilitaba la representación de partidos minoritarios, evitando que el partido mayoritario lograra un control absoluto de los escaños en el Congreso. Pero como discutimos a continuación, la estructura de incentivos del sistema binominal terminó por privilegiar tanto el alejamiento de los partidos del centro (ausencia de incentivos centrípetos) -comparado con lo que hubiera ocurrido de haberse adoptado un sistema uninominal- como la utilización del sistema electoral como un seguro contra la derrota electoral. Como mostramos a continuación, el sistema binominal no logra los objetivos del diseñador electoral pues no constituye un sistema electoral mayoritario, no contribuye a la formación de un sistema bipartidista, no favorece la moderación de todos los actores políticos ni evita que se reproduzca la nefasta experiencia electoral y partidista del pasado (Historia de la Ley 18.799, 39.) Es más, sugerimos que, además, el sistema binominal constituye un seguro contra la derrota, al permitir convertir una minoría electoral en una representación del $50 \%$ de los escaños en cada distrito.

Pese a que algunos han optado por definirlo como un sistema semimayoritario (Fernández, 1998), resulta evidente que el binominal es un sistema de representación proporcional donde cada entidad electoral posee una magnitud de distrito de dos escaños (Jones, 1995; Magar, Rosenblum y Samuels, 1998). La única diferencia con el sistema de representación proporcional existente antes de 1973 radica en el número de escaños que escoge cada distrito. Mientras antes del quiebre democrático, la gran mayoría de los distritos escogía un número igual o superior a los 3 escaños (salvo dos distritos que al escoger dos escaños cada uno constituían distritos binominales), el sistema actual establece que todos los distritos escogen el mismo número de legisladores, dos escaños por distrito.

Respecto al efecto reductivo en el sistema de partidos, si el objetivo del diseñador electoral hubiera sido producir un sistema bipartidista, la elección natural hubiera sido la adopción de un sistema uninominal. Pero como queda en evidencia en el Cuadro 1, que simula los resultados electorales entre 1989 y el 2001 suponiendo distritos uninominales, la Concertación hubiera obtenido la primera mayoría en un número sustancial de distritos en todas las elecciones. Los resultados del plebiscito de 1988 también reflejan un predominio de la Concertación. Aunque el No derrotó al Sí sólo en 151 de las 335 comunas del país (45\%), ellas congregaban al 77\% de los 7,4 millones de electores inscritos. En 1989, el candidato presidencial de la Concertación logró la victoria en 209 comunas (69\%). Si hubieran existido 120 distritos uninominales de un número relativamente comparable de electores, la Concertación habría logrado la victoria en alrededor de 80 a 100 distritos, suponiendo que éstos hubieran sido diseñados sin intención de sobre-representar a los partidos de derecha. Un sistema uninominal en las parlamentarias de 1989 hubiera producido una abrumadora victoria para la Concertación. 


\section{Cuadro 1}

Simulación de resultados, con 60 distritos uninominales, 1989-2001

\begin{tabular}{lccccccc}
\hline \# Distritos donde coalición ganadora obtuvo & primera mayoría de votos acumulados \\
\hline & $\mathbf{1 9 8 8}$ & $\mathbf{1 9 8 9}$ & $\mathbf{1 9 9 3}$ & $\mathbf{1 9 9 7}$ & $\mathbf{1 9 9 9}$ & $\mathbf{2 0 0 0}$ & $\mathbf{2 0 0 1}$ \\
\hline Concertación & 45 & 55 & 53 & 48 & 32 & 48 & 36 \\
Alianza & 15 & 4 & 17 & 12 & 28 & 12 & 23 \\
Otros & - & 1 & - & - & - & - & 1 \\
Total & 60 & 60 & 60 & 60 & 60 & 60 & 60 \\
\hline
\end{tabular}

Fuente: Cálculos de los autores con datos de http: / / www.elecciones.gov.cl

De haberse utilizado un sistema mayoritario, la Concertación habría logrado esa mayoría incontrarrestable que temía el diseñador electoral. Aunque, por otra parte, el tamaño de los distritos hubiera disminuido y las coaliciones se hubieran visto obligadas a presentar candidatos moderados que fueran capaces de obtener una mayoría de votos. Pese a no reconocerlo en la Historia de la Ley 18.799, la predominancia de la Concertación fue una de las razones por las que el gobierno militar optó contra un sistema mayoritario. Pero es importante señalar que dicha predominancia también constituye un resultado de la adopción del sistema binominal. Ya que no es preciso obtener una mayoría de votos para asegurarse un número satisfactorio de escaños en cada distrito, la coalición de derecha no tiene incentivos para buscar una votación mayoritaria. El binominal elimina los incentivos para buscar apoyo mayoritario en cada distrito.

Respecto al efecto moderador en el sistema de partidos, el binominal tiene incentivos estructurales que llevan a la polarización más que a la convergencia hacia el votante mediano (Magar, Rosenblum y Samuels, 1998.) La Figura 1 muestra los incentivos centrífugos del sistema binominal, comparados con los incentivos centrípetos del sistema uninominal. Mientras que en el uninominal un candidato necesita obtener una mayoría absoluta de los votos para asegurar el escaño, en el binominal basta con un $1 / 3+1$ de los votos. Con esa cantidad se puede obtener un $50 \%$ de los escaños en el distrito. Al igual que otros sistemas de representación proporcional, los incentivos para la polarización, asociados con una barrera de entrada menor que en los sistemas uninominales, quedaron enraizados en el Artículo 45 de la Constitución que establece la elección concurrente de dos senadores por región. Es cierto que al compararse con el sistema utilizado antes de 1973, la barrera de entrada es más alta en la Constitución de 1980. Mientras que antes bastaba con un 16,7\% de la votación para asegurarse un escaño senatorial, ahora basta con un tercio de la votación para lograrlo. Pero antes de 1973 se requería más del 50\% de la votación para asegurarse, unilateralmente, una mayoría de 
escaños en cada circunscripción senatorial, mientras que a partir de 1980 basta con un tercio de los escaños para evitar que alguien pueda obtener una mayoría de los escaños en cada circunscripción.

Figura 1

Incentivos centrífugos del sistema binominal

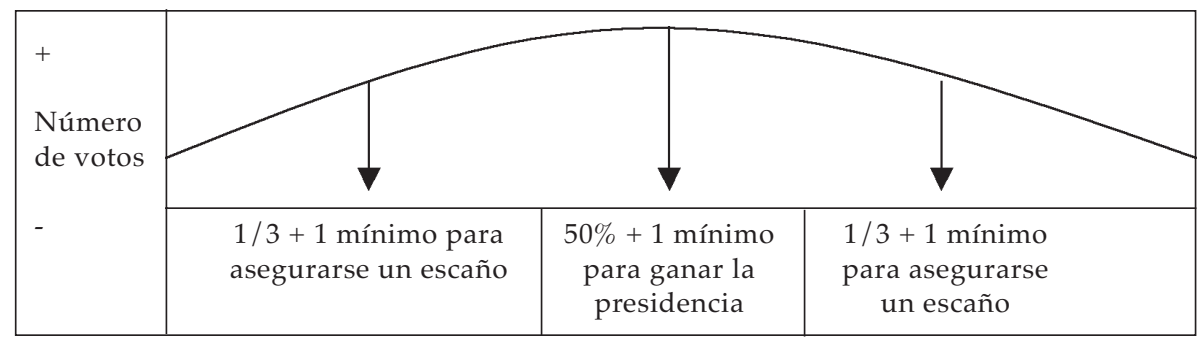

Fuente: elaboración propia.

Ahora bien, como acertadamente señala Jones (1999), cuando las elecciones presidenciales y parlamentarias se celebran en forma concurrente, la presencia de candidatos presidenciales contribuye a disminuir la proliferación de candidatos y partidos en las contiendas legislativas. La simultaneidad de las contiendas presidenciales y legislativas contribuye a aminorar el efecto multiplicador que tiene un sistema de representación proporcional en el número de candidatos y partidos que compiten. En ese sentido, bien pudiera argumentarse que la disminución en el número de partidos que compiten en Chile después de 1989 respecto a lo que ocurría antes de 1973 tiene más que ver con la simultaneidad de las contiendas presidenciales y legislativas que con la adopción de un sistema electoral diferente (eso lo discutimos en detalle más abajo).

Por los incentivos que fomenta el sistema binominal, sugerimos que constituye un seguro contra la derrota electoral. A diferencia de los distritos uninominales, donde el ganador se lo lleva todo, en sistemas de RP el costo de una derrota no significa perder todos los escaños en cada distrito. Mientras mayor sea la magnitud de distrito, mayor es la posibilidad de obtener escaños, aun si el partido obtiene una baja votación. En el caso de Chile es evidente, como señalan las propias citas de la comisión redactora de la ley, que la instancia legislativa rechazó la opción de distritos uninominales precisamente porque esperaba un apoyo electoral minoritario. Pero no basta con lograr algunos escaños en el Legislativo. La intención de aquél que escoge la ley electoral es lograr un número suficiente de escaños para ejercer una posición de veto a las iniciativas legislativas de la mayoría.

Al escoger una magnitud de dos escaños por distrito, el gobierno militar hizo muy difícil que un partido pudiera convertir una mayoría de votos en una mayoría de escaños en cada distrito. Como muestra la Figura 2, un partido necesita obtener más de 2/3 de los votos para asegurar am- 
bos escaños en el sistema binominal. Ahora bien, si hay más de dos partidos (o coaliciones) en competencia, la barrera para asegurar los dos escaños disminuye, en la medida que la votación que no va al partido mayoritario se divide en más de un partido de oposición. Pero si uno desconoce el número de partidos que participarán en una contienda electoral, lo más seguro es asumir que todos los votos que no vayan al partido del diseñador electoral irán a un solo partido de oposición.

Una fórmula simple permite identificar la barrera de votos que debe superar un partido mayoritario para lograr una mayoría de los escaños en cada distrito. Suponiendo una distribución de escaños utilizando la cifra repartidora d'Hondt, la fórmula para identificar la cantidad de votos sobre la cual un solo partido se asegura la mayoría de los escaños en cada distrito, independientemente de cuántos otros partidos compitan, es la siguiente:

\section{Cuando MD es un número par: \% Votos $>(100 M D+2) /(2 M D+2)$ \\ Cuando MD es un número impar: $\%$ Votos $>50 \%$}

Cuando el número de escaños es impar, basta con obtener una mayoría absoluta de votos para asegurarse una mayoría absoluta de los escaños en sistemas de representación proporcional. Pero cuando el número de escaños es par, la barrera mínima de votos para asegurarse una mayoría de los escaños en el distrito aumenta a medida que disminuye la magnitud del distrito. Así, la barrera más alta existe precisamente en distritos de dos escaños. Un partido debe obtener más de $2 / 3$ de los votos para, unilateralmente, asegurarse la mayoría de los escaños en el distrito. Más que buscar dañar a los partidos de izquierda o sobre-representar a los partidos de derecha, el gobierno militar buscó hacer particularmente difícil la posibilidad de transformar una mayoría electoral en un control mayoritario en el número de escaños en cada distrito.

Así, el sistema fue diseñado como un seguro contra las mayorías. Como muestra la Figura 2, cuando la magnitud de distrito es 2, la posibilidad de transformar una mayoría electoral en una mayoría en el número de escaños es más difícil que bajo cualquier otra magnitud de distrito, usando cualquier cifra repartidora. Si el que determina las leyes electorales no tiene certeza sobre el apoyo popular para su propio partido, puede escoger leyes que obstaculicen a cualquier partido obtener una mayoría de los escaños, aun si ese partido posee una mayoría de votos. Por cierto, en la medida que aumenta la magnitud de distrito, la barrera de votos necesaria para transformar una mayoría electoral en un control mayoritario de escaños disminuye. Así, si la magnitud de distrito es de 4 escaños, el mínimo de votos necesarios para controlar una mayoría de los escaños (3 escaños) es de $60 \%$. Si en cambio la magnitud del distrito es 8 , un partido asegura una mayoría de los escaños (5) con un $55,6 \%$ de la votación. 
Figura 2

$\%$ votos necesarios para obtener mayoría absoluta en un distrito (independiente de la votación de otros partidos)

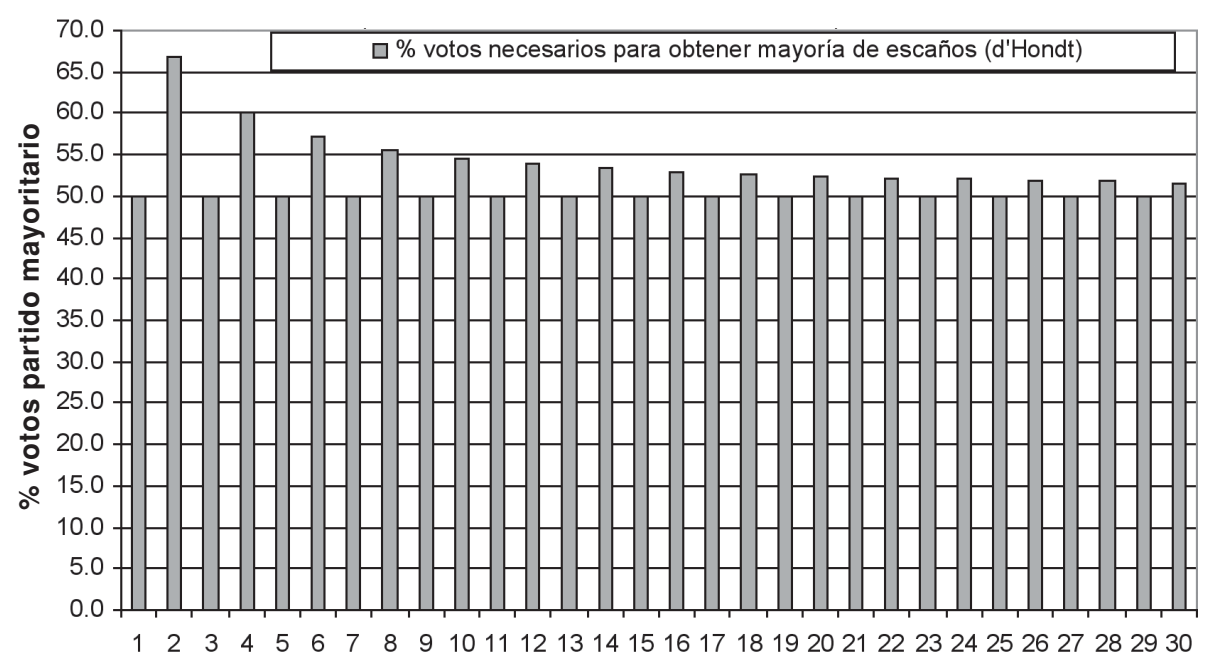

Magnitud de distrito

Fuente: elaboración propia.

Los resultados de las elecciones al Senado presentan evidencia concluyente de cómo funciona este seguro contra la derrota. En todas las elecciones celebradas desde 1989, la coalición de derecha recibió en promedio un porcentaje de escaños que fue superior en un $8,6 \%$ en promedio a su porcentaje de votos. A su vez, la Concertación recibió en promedio un porcentaje de escaños que fue superior en un $1,1 \%$ a su votación nacional. Así pues, el efecto de distorsión del sistema electoral, con su evidente diseño para evitar premiar con escaños adicionales a la coalición más votada constituye un eficiente sistema de seguro contra una derrota electoral. Pese a haber promediado solamente un $38,2 \%$ de los votos en las cuatro elecciones senatoriales celebradas en el país, la Alianza por Chile logró obtener un promedio de $46,8 \%$ de los escaños al Senado. Esto significa que la Alianza logró un 22,5\% más de escaños que de votos en el período ${ }^{3}$. La Concertación, en cambio, con un promedio de $52,8 \%$ de votos, logró un promedio de $53,2 \%$ de los escaños en la Cámara Alta. La diferencia entre votos y escaños para la Concertación fue mínima, mientras que para la derecha la votación de ese sector se vio sustancialmente sobre-representada en el porcentaje de escaños recibidos por el sector.

3. La diferencia entre el $38,2 \%$ de votos y el $46,8 \%$ de escaños representa el $22,5 \%$ de bonificación sobre la votación obtenida por ese sector. 


\section{Cuadro 2}

Resultados y asignación de escaños, elecciones senatoriales 1989-2001

\begin{tabular}{|c|c|c|c|c|c|c|c|c|}
\hline & \multicolumn{2}{|c|}{1989} & \multicolumn{2}{|c|}{1993} & \multicolumn{2}{|c|}{1997} & \multicolumn{2}{|c|}{2001} \\
\hline & $\begin{array}{r}\% \\
\text { votos }\end{array}$ & $\begin{array}{r}\% \\
\text { escaños }\end{array}$ & $\begin{array}{r}\% \\
\text { votos }\end{array}$ & $\begin{array}{r}\% \\
\text { escaños }\end{array}$ & $\begin{array}{r}\% \\
\text { votos }\end{array}$ & $\begin{array}{r}\% \\
\text { escaños }\end{array}$ & $\begin{array}{r}\% \\
\text { votos }\end{array}$ & $\begin{array}{r}\% \\
\text { escaños }\end{array}$ \\
\hline Concertación & 54,6 & 57,9 & 55,5 & 50 & 49,9 & 55 & 51,3 & 50 \\
\hline Alianza & 34,9 & 42,1 & 37,3 & 50 & 36,6 & 45 & 44,0 & 50 \\
\hline Otros & 10,5 & 0 & 7,2 & 0 & 13,5 & 0 & 4,7 & 0 \\
\hline Total & 100 & \#38 & 100 & $\# 18$ & 100 & \#20 & 100 & \#18 \\
\hline
\end{tabular}

Fuente: cálculos de los autores con datos de http:/ / www.elecciones.gov.cl

\section{El sistema binominal y la reducción en el número de partidos}

Después de haber destacado las características más salientes del sistema binominal, abordamos la pregunta inicial respecto a si éste ha contribuido, en la práctica, a disminuir el número de partidos que existen en el país. Para poder evaluar esto adecuadamente, utilizamos una conocida herramienta que mide el número de partidos políticos y candidatos por distrito. Optamos por hacerlo a nivel de distrito en vez de a nivel nacional siguiendo las recomendaciones de Cox (1997), quien argumenta que los efectos reductivos de las leyes electorales deben ser analizados a nivel local más que a nivel nacional.

Pero para evaluar adecuadamente el número de partidos y candidatos que ha existido en cada elección, en cada distrito debemos utilizar herramientas que se hagan cargo del peso electoral relativo diferente de los partidos y candidatos. Si midiéramos solamente el número de partidos y candidatos en competencia en cada distrito, no podríamos evaluar adecuadamente el peso electoral relativo de cada uno. Así, por ejemplo, no podríamos distinguir la diferencia entre dos distritos con 6 candidatos cada uno; pero en un distrito dos candidatos concentran el $80 \%$ de la votación mientras que en el otro cada candidato obtiene una votación cercana al 15\%. Aunque en ambos casos tenemos 6 candidatos, el distrito donde los dos candidatos con más votación concentran el $80 \%$ de los sufragios debiera ser considerado como con un menor número de candidatos que aquél donde los 6 candidatos se distribuyen equitativamente la votación. Por esa razón, en vez de medir el número absoluto de candidatos y partidos por distrito, evaluamos el número efectivo de partidos (NEP) y el número efectivo de candidatos (NEC). El método más conocido y utilizado para medir NEP y NEC fue propuesto por Laakso y Taagapera (1979; ver también Taagepera y Shugart, 1989). La fórmula utilizada es la siguiente:

$N E P=\left(\sum p_{i}\right)^{-1}$ 
NEP se obtiene al sumar el peso relativo (proporción de votos al cuadrado $-p_{i}{ }^{2}$ ) de cada uno de los partidos (o candidatos) en cada uno de los distritos. Para obtener un valor en número entero que permita simular el número efectivo de partidos que existen en cada distrito, se procede a elevar a ${ }^{-1}$ el valor obtenido. Este procedimiento lo utilizamos para medir el NEP y NEC para cada uno de los 60 distritos para las elecciones a la Cámara de Diputados realizadas desde 1989 al 2001. Para efectos de reportar más sucintamente nuestros resultados, utilizamos los valores promedios para los 60 distritos en cada elección. Debido a que nuestra intención es evaluar si el sistema electoral binominal contribuye a disminuir el número de partidos en competencia, realizamos el análisis midiendo tanto NEP como NEC. Ya que los partidos políticos en Chile compiten agrupados en coaliciones, una forma efectiva de medir las diferencias entre partidos y coaliciones es simplemente ignorando la militancia partidaria de los candidatos para evaluar el número de candidatos y peso relativo de cada uno de ellos, en cada uno de los distritos del país, en las cuatro elecciones que han sido celebradas desde 1989 hasta el 2001.

\section{Resultados: NEC y NEP}

Nuestro primer análisis consistió en medir tanto el número efectivo de candidatos (NEC) como el número efectivo de partidos (NEP) para todas las elecciones legislativas desde 1989 hasta el 2001 en cada uno de los 60 distritos electorales del país. El Cuadro 3 nos muestra que el NEP se mantuvo estable entre 1989 y el 2001. Mientras el NEP fue de 3,95 en 1989, en 1993 cayó a un 3,75. En 1997 subió a un 3,93 para volver a caer a 3,71 el 2001. Ninguna de esas diferencias en los valores del NEP es estadísticamente significativa (pruebas $t$ para una muestra) ${ }^{4}$. El Cuadro 3 nos muestra que podemos decir con un $95 \%$ de confianza que los datos de 1989, 1993, 1997 y 2001 comparten parte del rango de valores posibles - por lo tanto no podemos decir con certeza estadística que sean diferentes. Ya que todos los valores reportados en el Cuadro 3 caben dentro del rango del promedio para el período, podemos decir con suficiente certeza que no existe diferencia significativa del NEP medido a nivel distrital entre 1989 y 2001.

\footnotetext{
4. Aunque las pruebas $t$ para una muestra se utilizan normalmente para medir muestras y no diferencias de medias de distintos universos, si suponemos que el número efectivo de partidos y candidatos observados en una elección son muestras del número efectivo de candidatos y partidos que existe realmente en el electorado, entonces las pruebas t para una muestra son un instrumento adecuado.
} 


\section{Cuadro 3}

Número efectivo de candidatos y partidos políticos, 1989-2001

\begin{tabular}{lcccc}
\hline Año & $\begin{array}{c}\text { Número } \\
\text { efectivo }\end{array}$ & $\begin{array}{c}\text { Desviación estándar } \\
\text { (60 distritos) }\end{array}$ & Intervalos de 95\% de confianza \\
\hline NEP & 3.95 & 0,5765 & 3,8032 & 4,1011 \\
1989 & 3,75 & 0,5176 & 3,6143 & 3,8817 \\
1993 & 3,93 & 0,7156 & 3,8263 & 4,2096 \\
1997 & 3,71 & 0,5491 & 3,5801 & 3,8719 \\
2001 & 3,84 & 0,6003 & 3,7604 & 3,9130 \\
$1989-01$ & & 0,6746 & 4,0280 & 4,3764 \\
NEC & 4.20 & 0,5378 & 3,6072 & 3,8622 \\
1989 & 3,79 & 0,7423 & 3,7489 & 4,1126 \\
1993 & 4,01 & 0,5591 & 3,7495 & 4,1341 \\
1997 & 3,73 & 0,6572 & 3,8527 & 4,0198 \\
2001 & 3,94 & 0 & & \\
$1989-01$ & & 0 & & \\
\hline
\end{tabular}

Fuente: cálculos de los autores con datos de www.elecciones.gov.cl

Ahora bien, pese a no ser estadísticamente significativa, se evidencia una disminución entre 1989 y 1993. La razón principal que explica esa caída tiene que ver tanto con la ausencia de candidatos a la reelección en 1989 -tema que abordamos más adelante- como con la multiplicidad de partidos que existía en 1989, producto de la existencia de leyes que hacían ilegal a los partidos de orientación marxista. Hasta la aprobación de la reforma constitucional de mediados de 1989, el Partido Comunista y el Partido Socialista estaban proscritos (por lo que no tuvieron suficiente tiempo para constituirse en partidos políticos legales antes de las elecciones de diciembre). En 1989 no se presentaron candidatos comunistas a la elección parlamentaria. Los candidatos socialistas que se presentaron lo hicieron o bien como miembros del PPD o como independientes dentro del pacto Concertación. La multiplicidad de partidos en 1989 respondió en buena parte a las trabas legales que existían para que se inscribieran los socialistas y comunistas más que a los incentivos del sistema binominal. Por eso, el número efectivo de partidos en 1989 fue mayor al observado en elecciones posteriores. Pero queda claro que, contrario a lo que se hubiera esperado, la adopción del sistema binominal no contribuyó a una reducción de partidos a partir de 1989. 
Es cierto que dado que el sistema binominal fue adoptado con anterioridad a las elecciones de 1989, los partidos existentes habrían podido realizar ajustes para fusionarse antes de que celebraran las primeras elecciones. Pero sabemos que eso no fue así. Los partidos de la Concertación no optaron por hacerlo, sino que simplemente acordaron un sistema de omisiones y abstenciones que permitiera a ese conglomerado presentar sólo dos candidatos por distrito. Esto fue posible debido a que la LOC Sobre Votaciones Populares y Escrutinios fue modificada a mediados de 1989 por la Ley 18.799 (que, como ya discutimos, estableció el sistema binominal y determinó los límites de los 60 distritos para la Cámara de Diputados) permitiendo la formación de pactos electorales (artículo 3 Bis, Ley 18.700, introducido a través de la Ley 18.799 del 26 de mayo de 1989). Esta modificación a la Ley Electoral fue introducida, reconocidamente, para beneficiar a los dos grandes partidos de derecha, Renovación Nacional (RN) y la Unión Demócrata Independiente (UDI), que se habían trenzado en una dura disputa en 1988 que, entre otras cosas, terminó por romper el partido derechista unificado (RN) formado en 1987. Como era más probable que la Concertación se convirtiera en un partido a que lo hiciera la derecha, la dictadura optó por modificar la ley electoral para permitir la formación de pactos electorales (Allamand, 1999). Dicha decisión eliminó uno de los componentes de la LOC más conducentes a evitar la proliferación de partidos en el sistema político chileno: la prohibición de formar coaliciones electorales multipartidistas.

Lógicamente, la formación de pactos electorales contravenía el espíritu de la ley electoral que buscaba reducir el número de partidos políticos en el país. En la medida que la ley fue modificada para autorizar la formación de pactos electorales, era evidente que los partidos existentes tenderían a formar dichos pactos para evitar fusionarse con otros partidos afines. Aunque, como queda en evidencia en el Cuadro 3, la formación de pactos fue menos efectiva en 1989 que en 1993, pero fue tan efectiva ese primer año como lo sería en 1997 o el 2001. Ya que en 1989 se presentaron 7 pactos electorales además de varias candidaturas independientes fuera de pacto, las dos coaliciones más grandes (Concertación y pacto RN-UDI Democracia y Progreso) congregaron sólo el 85,6\% de la votación nacional. En 1993, totalizaron 92,1\%, en 1997 llegaron a un $86,7 \%$ y el 2001 alcanzaron el 92,2\%. Por otro lado, la Concertación se abstuvo de presentar un segundo candidato en muchos distritos donde la coalición de izquierda PAIS llevaba candidatos propios. Las tres candidaturas alternativas de partidos de derecha que se presentaron (Partido del Sur, Alianza del Centro y Liberal-Socialista Chileno) congregaron en conjunto un 6,35\% de la votación.

Porque hubo un total de 13 partidos, un grupo de independientes en cada uno de los 7 pactos nacionales y un pacto adicional de independientes (independientes fuera de pacto), el promedio nacional de partidos efectivos en 1989 fue de 3,95, el más alto observado en las cuatro elecciones parlamentarias celebradas hasta el 2001. Como mostramos más adelante, eso tuvo mucho que ver con la ausencia de titulares que se presen- 
taban a la reelección, lo que aumentaba el nivel de incertidumbre y hacía más difícil calcular el peso electoral relativo de los partidos en cada distrito. Por esa y muchas otras razones, las elecciones de 1989 se consideran como elecciones fundacionales -en la terminología de las transiciones a la democracia- y resulta peligroso compararlas con elecciones posteriores en condiciones de mayor normalidad democrática.

El NEP más bajo observado en las tres elecciones democráticas normales celebradas entre 1993 y 2001 fue el observado el 2001. Pero como señala el Cuadro 3, ese valor no es diferente -con un 95\% de confianza estadística- a los observados en las otras dos elecciones. Esto es, contrario al efecto reductivo esperado del sistema binominal, el NEP no se modificó sustancialmente después de 1993. Ahora bien, el hecho que las elecciones presidenciales de 1993 hayan sido celebradas en forma conjunta con la contienda parlamentaria bien pudiera contribuir a explicar (siguendo a Jones, 1999) la disminución en el NEP ese año respecto a 1989, y también el aumento en 1997 respecto a 1993. Pero la ausencia de elecciones presidenciales concurrentes a las legislativas el 2001 no llevó a una mayor dispersión de partidos. Esto es, la multiplicidad de partidos en Chile pareciera ser independiente a los incentivos institucionales -como la ley electoral o la presencia de elecciones presidenciales concurrentes- que existen en el país. Pese a tener valores del NEP inferiores a los observados en las elecciones antes de 1973, el sistema binominal no logró eliminar la presencia del multipartidismo en el sistema político chileno.

El Cuadro 3 también nos muestra que el número efectivo de candidatos (NEC), no obstante, tendió a disminuir entre 1989 y el 2001. Mientras esa cifra alcanzaba a 4,20 en 1989; para el 2001 alcanzó sólo a 3,73, demostrando una reducción que es estadísticamente significativa. De hecho, el NEC de 1989 también es estadísticamente diferente del NEC de 1993 (aunque no del de 1997). La caída del NEC con posterioridad a 1989 refleja, más allá de toda duda, una disminución real en el número de candidatos. Como argumentamos también más abajo, esto tiene que ver más con la ausencia de diputados titulares que se presentaban a la reelección en 1989 que con el efecto disuasivo del sistema electoral. El NEC de 1989 es superior, y estadísticamente distinguible de los valores de 1993 y 2001, y también superior (aunque no con confianza estadística) al de 1997. Esto quiere decir que después de 1989 el NEC se ha estabilizado en un rango de valores sustancialmente inferior. Nuevamente, este resultado tiende a validar la tesis que la verdadera razón que explica la disminución en el NEC tiene más que ver con la presencia de candidatos a la reelección que con los efectos del sistema binominal. Como no había candidatos a la reelección en 1989, simplemente se presentaron más candidatos que en elecciones posteriores.

Ahora bien, intuitivamente esperaríamos que el NEC fuera superior al NEP. Esto porque cada partido pudiera presentar más de un candidato en un determinado distrito, dividiendo así la votación partidista y contribuyendo a que haya un número de candidatos relevantes superior al 
número de partidos relevantes. Este supuesto resulta claramente cierto para las parlamentarias de 1989 (donde el NEC fue de 4,20 y el NEP de 3,95), pero para las parlamentarias de 1993, 1997 y 2001 la diferencia superior de NEC sobre NEP es mínima (3,79 sobre 3,75 en 1993, 4,02 sobre 3,93 en 1997 y 3,73 sobre 3,71 el 2001). Esto demuestra que la tendencia creciente de los partidos en el país es a presentar un solo candidato por distrito. Aunque en todas las elecciones hay distritos donde los partidos presentan más de un candidato, la tendencia creciente es a que cada partido tenga como máximo un candidato por distrito. Cuando eso ocurra en todo el país, los valores del NEC y NEP serán idénticos.

Ahora bien, los defensores del sistema binominal argumentarían que, dada la lógica de coaliciones, el sistema contribuye a la reducción en el número de coaliciones que se presentan a una elección. Pero tanto el NEP como el NEC de las últimas tres parlamentarias (1993-2001) nos muestra una tendencia a la estabilización en torno a cuatro partidos y cuatro candidatos efectivos en cada distrito para cada elección. Parecieran tener razón los críticos del sistema binominal que insisten en que el sistema debe ser entendido como si contribuyera a una lógica bipartidista al interior de las dos grandes coaliciones. En cada distrito tiende a darse una lógica en la que compiten esencialmente cuatro candidatos de cuatro partidos diferentes. Si bien la presencia de las dos grandes coaliciones pareciera ser compatible con el sistema electoral, la permanencia de cuatro partidos fuertes también pareciera ser una consecuencia inevitable de este sistema supuestamente diseñado para reducir el número de partidos políticos en el país.

\section{Resultados: Titulares buscando la reelección}

Como ha quedado en evidencia en el Cuadro 3, el NEC y el NEP son superiores en 1989 que en las tres elecciones posteriores. Argumentamos que aquello -especialmente el valor superior del NEC- es resultado de la ausencia de candidatos que se presentaban a la reelección. Aportando evidencia adicional que apoya dicha aseveración, el Cuadro 4 nos muestra los resultados del NEC y NEP para los distritos agrupados de acuerdo al número de titulares que se presentan a la reelección entre 1993 y el 2001. Naturalmente, en 1989 no podían presentarse titulares a la reelección, por lo que excluimos esos 60 distritos de la muestra. De tal forma que quedamos sólo con 180 distritos, para tres elecciones al Legislativo. Aunque el número total de distritos donde ningún candidato se presentó a la reelección es bajo (13), y por lo tanto es extremadamente difícil encontrar patrones estadísticamente significativos, el Cuadro 4 deja en evidencia que tanto el NEC como el NEP son superiores para los distritos donde no se presenta ningún titular a reelección que en distritos donde se presenta uno o dos titulares a la reelección. Es más, en aquellos distritos donde se presentan dos titulares a la reelección, los valores del NEP y NEC son aún más bajos que en distritos donde se presenta sólo 
un titular. Aunque esas diferencias tampoco son estadísticamente significativas, las tendencias van en la dirección esperada. Mientras más candidatos se presentan a la reelección, menor es el valor del NEP y NEC.

\section{Cuadro 4}

NEP y NEC según presencia de candidatos titulares a reelección, 1993-2001

\begin{tabular}{lccc}
\hline $\begin{array}{l}\text { Número de titulares a } \\
\text { reelección por distrito }\end{array}$ & Número de distritos & NEP & NEC \\
\hline 0 & 13 & 4.04 & 4.19 \\
1 & 76 & 3.82 & 3.89 \\
2 & 91 & 3.74 & 3.76 \\
Total & 180 & 3.80 & 3.85 \\
\hline
\end{tabular}

Fuente: cálculos de los autores con datos de www.elecciones.gov.cl

Al desagregar los resultados elección a elección, encontramos -Cuadro 5- que en 1993 los valores de NEC y NEP son superiores en los distritos donde no se presentan titulares a la reelección. En 1997 y 2001, el número de dichos distritos es reducido ( 3 y 2, respectivamente). Pero en ambos años los valores de NEC y NEP son superiores en los distritos donde se presenta un candidato a la reelección que en aquellos distritos donde se presentan dos candidatos a la reelección. Una vez más, la reducción en los valores de NEC observados en 1993, 1997 y 2001 respecto a lo observado en 1989 bien pudiera tener más que ver con la presencia de titulares que buscan la reelección -y que si no tienden a disuadir a potenciales rivales, al menos les hacen más difícil conseguir votos- que con los efectos reductivos que algunos atribuyen al sistema binominal.

Cabe destacar que la ausencia de suficientes distritos 'abiertos' (donde no se presenta ningún candidato a la reelección) en 1997 y 2001 hace imposible verificar si el alto valor de NEC observado en 1989 tiene una correlación directa con la ausencia de titulares. Pero la evidencia inicial es concluyente sobre una relación inversa entre el número de titulares que se presenta a la reelección y el NEC y NEP en las elecciones para la Cámara de Diputados a nivel de distritos. Mientras menos titulares se presentan a la reelección, más aumenta el número de candidatos que consiguen votación considerable. Inversamente, mientras más titulares se presentan a la reelección, más se congrega la votación en un número inferior de candidatos. Esto sustenta la tesis que la disminución de candidatos y partidos en las elecciones legislativas con posterioridad a 1989 se explica fundamentalmente por la presencia disuasiva de diputados titulares. Esto ya sea porque simplemente se presentan menos candidatos a los puestos o porque, de presentarse, simplemente los electores concentran su votación en los candidatos titulares que buscan la reelección. 


\section{Cuadro 5}

Número de titulares a reelección por distrito, 1989-2001

\begin{tabular}{|c|c|c|c|}
\hline $\begin{array}{l}\text { Número de titulares } \\
\text { por distrito }\end{array}$ & Número de distritos & NEP & NEC \\
\hline \multicolumn{4}{|l|}{1989} \\
\hline 0 & 60 & 3,95 & 4,20 \\
\hline 1 & - & 0 & 0 \\
\hline 2 & - & 0 & 0 \\
\hline Total & 60 & 3,95 & 4,20 \\
\hline \multicolumn{4}{|l|}{1993} \\
\hline 0 & 8 & 4,14 & 4,38 \\
\hline 1 & 18 & 3,52 & 3,56 \\
\hline 2 & 34 & 3,78 & 3,78 \\
\hline Total & 60 & 3,75 & 3,79 \\
\hline \multicolumn{4}{|l|}{1997} \\
\hline 0 & 3 & 3,98 & 4,00 \\
\hline 1 & 30 & 4,00 & 4,13 \\
\hline 2 & 27 & 3,86 & 3,90 \\
\hline Total & 60 & 3,93 & 4,02 \\
\hline \multicolumn{4}{|l|}{2001} \\
\hline 0 & 2 & 3,76 & 3,77 \\
\hline 1 & 28 & 3,83 & 3,85 \\
\hline 2 & 30 & 3,60 & 3,62 \\
\hline Total & 60 & 3.71 & 3,73 \\
\hline
\end{tabular}

Fuente: cálculos de los autores con datos de www.elecciones.gov.cl

\section{Resultados: Mujeres candidatas}

La presencia de candidatas mujeres está asociada con el NEC. Como lo muestra el Cuadro 6, existe una correlación inversa. A medida que aumenta el número de candidatas mujeres disminuye el valor NEC. En los 108 distritos en que no se presentó ninguna mujer, el NEC promedio fue de 3,99. En los 68 distritos en que se presentó una mujer, el valor promedio del NEC fue de 4,00. En los 49 distritos en que se presentaron dos mujeres a la elección, el valor promedio del NEC fue de 3,86. Existe una tendencia a la baja en el valor del NEC a medida que se presentan más mujeres. Esto quiere decir que mientras mayor sea el número de mujeres, más se concentra la votación distrital en menos candidatos. Aunque este resultado amerita un estudio más profundo -y los datos no son estadísticamente significativos-, podemos anticipar algunas hipótesis plausibles. Tal vez los partidos nominan mujeres en distritos donde ya hay candidatos fuertes que van o no a la reelección y donde las posibilidades de las 
mujeres de salir electas son relativamente bajas. Inversamente, podríamos también sugerir que los partidos nominan mujeres en distritos donde su votación es relativamente fuerte -para así maximizar las posibilidades de que salgan electas- y por lo tanto los bajos valores del NEC reflejan sólo la fortaleza de los partidos en dichos distritos.

\section{Cuadro 6}

Presencia de candidatas por distrito, todas las elecciones de 1989 a 2001

\begin{tabular}{lccc}
\hline $\begin{array}{l}\text { Número de } \\
\text { candidatas mujeres }\end{array}$ & Número de distritos & $\begin{array}{c}\text { Número efectivo } \\
\text { de partidos (NEP) }\end{array}$ & $\begin{array}{c}\text { Número efectivo } \\
\text { de candidatos (NEC) }\end{array}$ \\
\hline 0 & 108 & 3,83 & 3,99 \\
1 & 68 & 3,89 & 4,00 \\
2 & 49 & 3,83 & 3,86 \\
3 & 10 & 3,71 & 3,75 \\
4 & 5 & 3,65 & 3,68 \\
Total & 240 & 3,84 & 3,94 \\
\hline
\end{tabular}

Fuente: cálculos de los autores con datos de www.elecciones.gov.cl

Por otro lado, no se observa una asociación significativa entre el número de mujeres y el NEP. Dado que un mayor número de candidatas aparece asociado con partidos políticos que generalmente no logran escaños en el Parlamento -PC y PH-, bien pudiéramos estar observando sólo el resultado de la presencia de candidatas de partidos que no tenían muchas posibilidades iniciales de lograr ser electas y por lo tanto no producen ningún efecto en el NEP.

$\mathrm{Al}$ evaluar el NEP respecto a la cantidad de candidatas mujeres separadamente para las cuatro elecciones, tampoco logramos encontrar evidencia de cambios que sean estadísticamente significativos o que reflejen alguna posible tendencia. Esto fundamentalmente porque el número de distritos donde se presentan mujeres candidatas es relativamente bajo en cada elección. De hecho, en 200 de los 240 distritos analizados en las 4 elecciones, no se ha presentado ninguna candidata. Naturalmente, la poca presencia de candidatas hace difícil analizar las tendencias históricas, aunque la ausencia de mujeres subraya problemas de acceso que probablemente respaldan la tesis que los partidos estratégicamente nominan mujeres como candidatas, ya sea en lugares donde no tienen posibilidades de salir electas o bien en distritos seguros donde tienen altas posibilidades de hacerlo. 


\section{Cuadro 7}

Número efectivo de partidos y número de candidatas electas

\begin{tabular}{lcccccccc}
\hline Año & \multicolumn{7}{c}{ Número de mujeres electas } \\
\cline { 2 - 8 } & $\mathbf{0}$ & $\mathbf{N}$ & $\mathbf{1}$ & $\mathbf{N}$ & $\mathbf{2}$ & $\mathbf{N}$ & Total & $\mathbf{N}$ \\
\hline 1989 & 3,95 & 55 & 4,33 & 3 & 3,55 & 2 & 3,95 & 60 \\
1993 & 3,75 & 51 & 3,73 & 9 & NA & NA & 3,75 & 60 \\
1997 & 3,91 & 47 & 4,02 & 13 & NA & NA & 3,93 & 60 \\
2001 & 3,72 & 47 & 3,61 & 12 & 4,77 & 1 & 3,71 & 60 \\
TODOS & 3,84 & 200 & 3,84 & 37 & 3,96 & 3 & 3,84 & 240 \\
\hline
\end{tabular}

Fuente: cálculos de los autores con datos de www.elecciones.gov.cl

\section{¿Qué nos dice el NEC y el NEP sobre posibles reformas al sistema electoral?}

En la medida que el debate sobre una reforma al sistema binominal cobra fuerza, los resultados de nuestro estudio pueden contribuir a anticipar posibles efectos de cambios al sistema electoral que introduzcan mayor proporcionalidad al sistema. Como ha quedado en evidencia, la naturaleza multipartidista del sistema político chileno ha sobrevivido a los incentivos reductivos del sistema binominal. Después de 16 años de ser el sistema a través del cual los votos se transforman en escaños, el sistema de partidos chilenos continúa reflejando una naturaleza pluripartidista similar a la que existía hasta antes de 1973. Resulta, por lo tanto, difícil anticipar que una reforma que introduzca mayor proporcionalidad al sistema inevitablemente debería producir un aumento en el número de partidos políticos que existen en el país. Aunque inevitablemente un sistema más proporcional disminuiría las barreras de entrada para que partidos minoritarios alcanzaran representación en el Parlamento, la formación (y la desaparición) de partidos políticos en Chile pareciera tener que ver más con coyunturas sociales (los llamados cleavages, o fallas) que, exclusivamente, con modificaciones al sistema electoral.

Por otro lado, la evidencia es concluyente respecto al efecto reductivo en el número de partidos y candidatos que tiene el hecho que un porcentaje relativamente elevado de legisladores busque la reelección cada año. En la medida que una reforma electoral que haga más proporcional el sistema no incluya también límites a la cantidad de períodos que los legisladores pueden servir en el Parlamento, no deberíamos ver un aumento demasiado significativo en el número efectivo de partidos y candidatos en Chile. Pero si en cambio se adoptan reformas que limiten el número de períodos que los legisladores pueden permanecer en el legislativo, entonces deberíamos ver un aumento en el número efectivo de partidos y de candidatos, aun si se mantiene el sistema binominal. 
Por otro lado, la reforma constitucional que permite que, desde el 2005 en adelante, todas las elecciones presidenciales y parlamentarias sean simultáneas no debería tener mayores efectos en el número efectivo de partidos y candidatos, de mantenerse el sistema binominal. Pero bien pudiera ser, como sugiere la literatura, que de adoptarse un sistema electoral más proporcional, el hecho que las elecciones parlamentarias y presidenciales se celebren en forma conjunta, contribuirá a que el número efectivo de partidos y candidatos que compiten en las elecciones no aumente significativamente.

\section{Conclusiones}

En este trabajo no hemos encontrado evidencia que apoye la tesis que el sistema binominal haya logrado reducir el número de partidos o candidatos que se presentan a elecciones en la Cámara de Diputados. El binominal no contribuye a reducir el número efectivo de partidos políticos ni el número efectivo de candidatos. Aunque alegadamente el sistema fue adoptado para promover la formación de dos partidos que tendieran hacia el centro, los datos empíricos demuestran que tanto el NEP como el NEC se han mantenido estables desde que el sistema fue inicialmente adoptado en 1989. Hemos encontrado suficiente evidencia también para sugerir que la presencia de candidatos titulares que se presentan a reelección sí contribuye a reducir el NEC y el NEP que existen a nivel distrital. La existencia de la reelección parece tener un efecto más importante en reducir el número de candidatos y partidos en elecciones recientes que el sistema binominal. Finalmente, también hemos mostrado que la presencia de candidatas tiene una asociación negativa con el NEC, pero no se observa una asociación entre la presencia de candidatas y el NEP.

Basados en la evidencia discutida aquí, creemos que es importante incorporar el debate sobre los límites a la reelección de los parlamentarios a la discusión más general sobre el sistema electoral. De mantenerse el binominal, pero adoptarse límites a la cantidad de reelecciones que puede buscar un diputado, el número de partidos y candidatos bien pudiera aumentar y alcanzar los niveles observados en 1989. De la misma forma, de mantenerse las altas tasas de titulares que buscan la reelección, una modificación al sistema electoral hacia un mayor número de escaños por distrito -en especial si las elecciones presidenciales y legislativas se realizan siempre en forma concurrente- pudiera no alterar significativamente el número de partidos políticos efectivos que compiten en las contiendas electorales para la Cámara de Diputados.

De cualquier forma, creemos importante subrayar que no hemos encontrado evidencia que demuestre que los incentivos del sistema electoral hayan provocado una disminución en el número de partidos y candidatos que efectivamente compitieron en las elecciones para la Cámara de Diputados en el período comprendido entre 1989 y el 2001. 


\section{Bibliografía}

Allamand, Andrés. 1999. "Las paradojas de un legado". En: Paul Drake e Iván Jaksic (eds.), El modelo chileno. Democracia y desarrollo en los noventa. Santiago: LOM: 169-190.

Collier, Simon y Sater, William F. 1996. A History of Chile. 1808-1994. Cambridge: Cambridge University Press.

Cox, Gary. 1997. Making Votes Count. New York: Cambridge University Press.

Fernández, Mario. 1998. “El sistema político chileno. Características y tendencias". En Cristián Toloza y Eugenio Lahera (eds.), Chile en los noventa. Santiago, Presidencia de la República/DOLMEN: 27-52

Garretón, Manuel Antonio. 1991. “The Political Opposition and the Party System under the Military Regime". En: Paul Drake e Ivan Jaksic (eds.). 1995. The Struggle for Democracy in Chile. 1982-1990. Revised Edition. Lincoln: University of Nebraska Press.

Historia de la Ley 18799. Santiago: Biblioteca del Congreso.

Jones, Mark P. 1995. Electoral Laws and the Survival of Presidential Democracies. South Bend: University of Notre Dame Press.

Jones, Mark P. 1999. "Electoral Laws and the Effective Number of Candidates in Presidential Elections". Journal of Politics 61: 1.

Laakso, Marku y Taagapera, Rein. 1989. “Effective Number of Parties: A Measure with Application to West Europe". Comparative Political Studies 12: 3-27.

Libertad y Desarrollo. 16 de marzo de 2001. "La Constitución 20 años después". Temas Públicos 520.

Magar, Eric y Marc R. Rosenblum, David J. Samuels. diciembre 1998. "On the absence of centripetal Incentives in Double-Member Districts. The Case of Chile". Comparative Political Studies 31 (6): 714-739.

Nohlen, Dieter. 1994. Sistemas de partidos y sistemas electorales. México: Fondo de Cultura Económica.

Pastor, Daniel. 2004. "Origins of the Chilean Binominal Election System". Revista de Ciencia Politica Vol. XXIV (1): 38-57.

Portales, Felipe. 2000. Chile. Una democracia tutelada. Santiago: Sudamericana.

Siavelis, Peter. 1993. "Nuevos argumentos y viejos supuestos. Simulaciones de sistemas electorales alternativas para las elecciones parlamentarias chilenas". Estudios Públicos 51 (Invierno): 229-267.

Siavelis, Peter. 1997a. "Executive-Legislative Relations in Post-Pinochet Chile: A Preliminary Assessment". En: Scott Mainwaring y Matthew Soberg Shugart (eds.), Presidentialism and Democracy in Latin America. New York: Cambridge University Press.

Siavelis, Peter. 1997b. "Continuity and Change in the Chilean Party System". Comparative Political Studies 30 (6): 651-674.

Siavelis, Peter. 2000. The President and Congress in Post-Authoritarian Chile: Institutional Constraints to Democratic Consolidation: Penn State University Press.

Sigmund, Paul E. 1993. The United States and Democracy in Chile. Baltimore: Johns Hopkins University Press. 
Taagepera, Rein y Shugart, Matthew Soberg. 1989. Seats and Votes. The Effects and Determinants of Electoral Systems. New Haven: Yale University Press.

Valenzuela, Arturo y Siavelis, Peter. 1991. "Ley electoral y estabilidad democrática: un ejercicio de simulación para el caso de Chile". Estudios Públicos 43 (Invierno): $27-87$.

Fecha de recepción: Noviembre de 2005.

Fecha de publicación: Diciembre de 2005. 